\title{
LUNG ULTRASOUND DURING COVID-19 PANDEMICS: WHY, HOW AND WHEN?
}

DOI: 10.36740/WLek202108101

\author{
Khrystyna 0. Pronyuk', Liudmyla 0. Kondratiuk', Andrii D. Vysotskyi², Olga A. Golubovska', Iryna M. Nikitina ${ }^{3}$ \\ 'INFECTIOUS DISEASES DEPARTMENT, 0.BOGOMOLETS NATIONAL MEDICAL UNIVERSITY, KYIV, UKRAINE \\ ${ }^{2}$ OLEXANDRIVSKA CLINICAL HOSPITAL, KYIV, UKRAINE \\ ${ }^{3}$ SUMY STATE UNIVERSITY, SUMY, UKRAINE
}

\begin{abstract}
The aim: To optimize diagnostic of pathological processes in lungs affected by COVID-19, dynamic monitoring and clinical decision making using lung ultrasound in limited resources settings.

Materials and methods: Between the onset of pandemics and January 2021, approximately 9000 patients have been treated for confirmed COVID-19 in the 0lexandrivska Clinical Hospital. Assessment of all hospitalized patients included hematology, chemistries and proinflammatory cytokines - IL-6, CRP, procalcitonin, ferritin. Diagnosis was confirmed by PCR for SARS-CoV-2 RNA. Chest X-ray was performed in all hospitalized cases, while CT was available approximately in 30\% of cases during hospital stay. Lung ultrasound was proactively utilized to assess the type and extent of lung damage and to monitor the progress of disease in patients hospitalized into the ICU and Infection Unit $(n=135)$. Ultrasound findings were recorded numerically based on scales.

Results: In the setting of COVID-19, bedside lung ultrasound has been promptly recognized as a tool to diagnose and monitor the nature and extent of lung injury. Lung ultrasound is a real time assessment, which helps determine the nature of a pathologic process affecting lungs. In this paper the accuracy of bedside LUS, chest X-ray and computer tomography are compared based on clinical cases, typical for COVID-19 lung ultrasound appearance is evaluated. Described in article data is collected in one of the biggest facility that deals with COVID-19. Chest X-ray was performed in all hospitalized cases, while CT was available approximately in 30\% of cases during hospital stay. The cases presented in the paper indicate potential advantages to the use of ultrasound in limited resource healthcare settings, especially when the risk of transportation to $\mathrm{CT}$ outweighs the value of information obtained.

Conclusions: Grading of ultrasonographic findings in the lungs was sufficient for both initial assessment with identification of high risk patients, and routine daily monitoring. Hence, lung ultrsound may be used to predict deterioration, stratify risks and make clinical decisions.
\end{abstract}

KEY WORDS: COVID-19, lung ultrasound, chest computed tomography, radiography, treatment strategy

Wiad Lek. 2021;74(8):1783-1788

\section{INTRODUCTION}

There is an urgent need for tools to adequately assess pathological processes in the lungs affected by COVID-19, especially in ventilated patients. Computed tomography $(\mathrm{CT})$ of the chest is seen as the gold standard to assess location, differentiation, and extent of pathologic processes affecting lungs, when chest X-ray is the most frequently used modality. Regretfully, both modalities imply transporting contagious patients, and require staff trained in mechanical ventilation and cardiopulmonary monitoring [1]. The cost of both modalities is high as well, which is even more relevant in the setting of pandemics with high number of daily assessments and frequent scarcity of resources. Moreover, while CT does provide adequate assessment of pathologic process and helps determine the phenotype of lung injury in a specific patient, chest X-ray, although more accessible, does not answer many questions. Bedside X-ray images are often of unsatisfactory quality and low sensitivity $[2,3]$. It has been shown that even when the assessment is strictly controlled, at least $30 \%$ of obtained images are suboptimal, and in some cases chest X-ray data poorly correlate with CT findings [2-4]. The very technique of $X$-ray imaging involves deep inspiration, which may cause small alveolar consolidations to disappear. Thus, a normal X-ray film may be obtained despite existing lung injury. These limitations cause inadequate and delayed assessment of lung injury in terms of type, degree and extent. This, in turn, may affect the management strategy including the choice of oxygen supplementation technique.

In the setting of COVID-19, bedside lung ultrasound has been promptly recognized as a tool to diagnose and monitor the nature and extent of lung injury [5]. Higher sensitivity of lung ultrasound versus chest X-ray has been shown for detection of pleural effusion, pulmonary consolidations, and interstitial syndrome in acute respiratory distress syndrome (ARDS) [1], justifying the use of this modality to assess the severity of lung injury in COVID-19. 

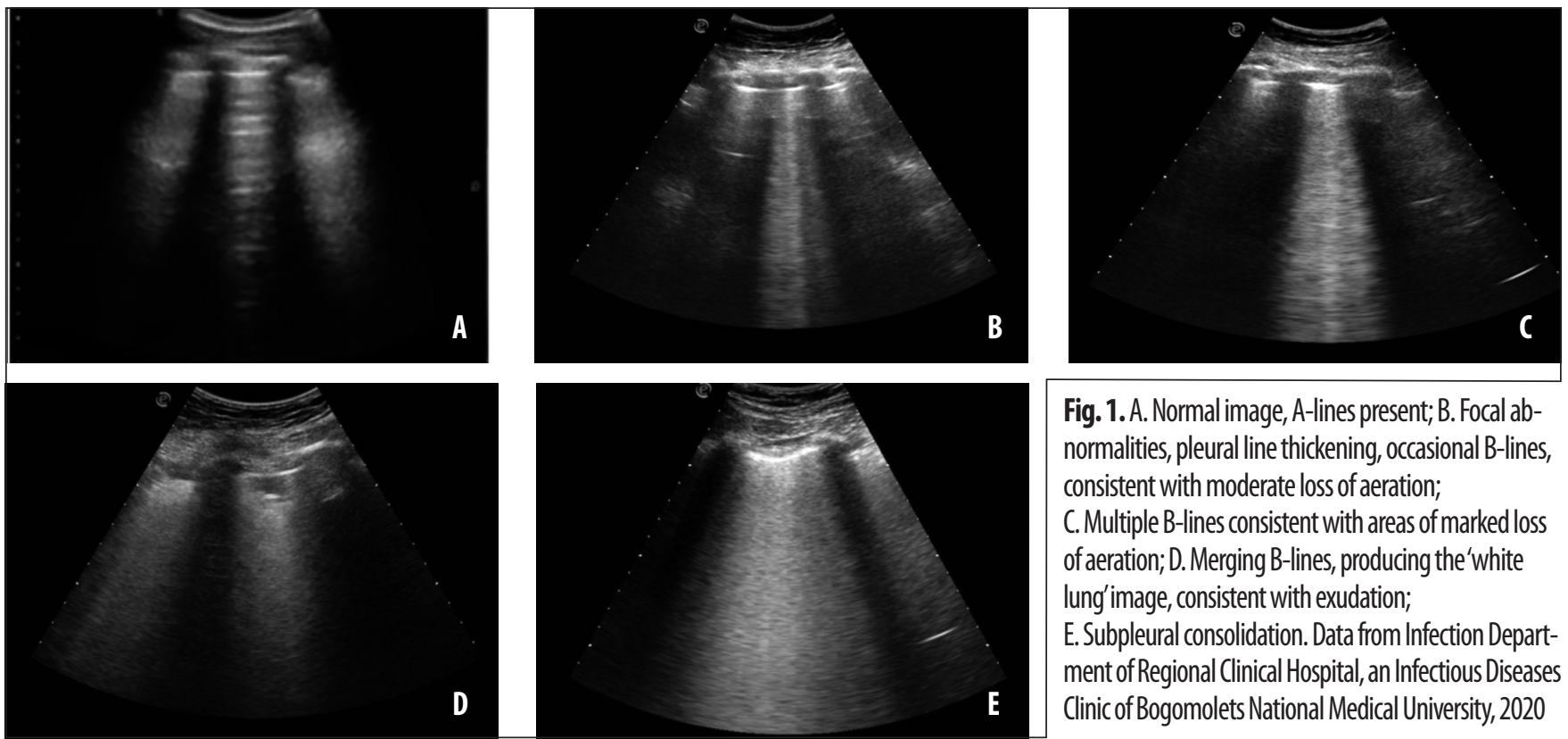

Fig. 1. A. Normal image, A-lines present; B. Focal abnormalities, pleural line thickening, occasional B-lines, consistent with moderate loss of aeration; C. Multiple B-lines consistent with areas of marked loss of aeration; D. Merging B-lines, producing the'white lung'image, consistent with exudation;

E. Subpleural consolidation. Data from Infection Department of Regional Clinical Hospital, an Infectious Diseases Clinic of Bogomolets National Medical University, 2020

\section{THE AIM}

To optimize diagnostic of pathological processes inlungs affected by COVID-19, dynamic monitoring and clinical decisionmaking using lung ultrasound in limited resources settings.

\section{MATERIALS AND METHODS}

Between the onset of pandemics and January 2021, approximately 9000 patients have been treated for confirmed COVID-19 in the Olexandrivska Clinical Hospital. Assessment of all hospitalized patients included hematology, chemistries and proinflammatory cytokines - IL-6, CRP, procalcitonin, ferritin. Diagnosis was confirmed by PCR for SARS-CoV-2 RNA. Chest X-ray was performed in all hospitalized cases, while CT was available approximately in $30 \%$ of cases during hospital stay. For some patients CT had been performed before hospital admission. Lung ultrasound was proactively utilized to assess the type and extent of lung damage and to monitor the progress of disease in patients hospitalized into the ICU and Infection Unit $(n=135)$. Ultrasound findings were recorded numerically based on scales.

\section{How? The technique}

Gas-filled anatomical structures are typically impenetrable to ultrasound, therefore lung parenchyma cannot be visualized below the pleural line [1]. However, a pathological process may change the aeration of pulmonary tissue, giving rise to a number of echogenic artifacts. Convex probe with abdominal settings is typically used, and no image filters are applied. The probe is placed perpendicular to the intercostal space, which comprises the acoustic window for lung tissue assessment. The following is seen on a normal ultrasound image:

1) Pulmonary sliding symptom

2) Presence of A-lines, hyperechoic horizontal artifacts produced by reverberation from the pleural line (Fig. 1A)
3) B-lines - hyperechoic vertical artifacts resembling 'comet tails' and originating from the pleural line, normally not more than 2 per intercostal space.

Increased number of B-lines is typical for interstitial syndrome. It has been shown that the so called 'lung rockets' correspond to thickening of alveolar septae, while 'white lung' (merging B-lines) correspond to ground glass opacity on CT [1,7-9]. In case of severe loss of aeration and alveolar consolidations adjacent to the pleura, the lung is seen as a parenchymatous structure containing hyperechoic artifacts - bronchograms, which correspond to bronchi in terms of morphology [6].

Lung ultrasound is a real time assessment, which helps determine the nature of a pathologic process affecting lungs. Regular assessments are useful as follow-up after procedures and to monitor changes in condition of patients. It should be noted that lung ultrasound may be used to select PEEP and other mechanical ventilation parameters (Fig. 2). Inability to detect hyperinflation is a drawback of the modality.

Classical lung ultrasound as part of POCUS, including the well-known BLUE protocol, is a quick and standardized problem-focused bedside algorhythm of limited scope based on the three standard points. However, a more extensive targeted assessment is required to determine the nature and extent of pulmonary abnormalities and for decision-making in COVID-19 setting. We use the algorhythm proposed by Italian physicians, which is based on imaging in 12 standard areas [10]. Each area is assessed for pleural lines, B-lines, and the presence of alveolar consolidations, and graded from 0 if the ultrasound pattern is normal to 3 if pulmonary consolidation is present. The total of area grades may be used to track clinical progress, whereby higher score is consistent with loss of pulmonary tissue aeration and clinical deterioration. If posterior segments are not accessible to examination in a critical patient on invasive 

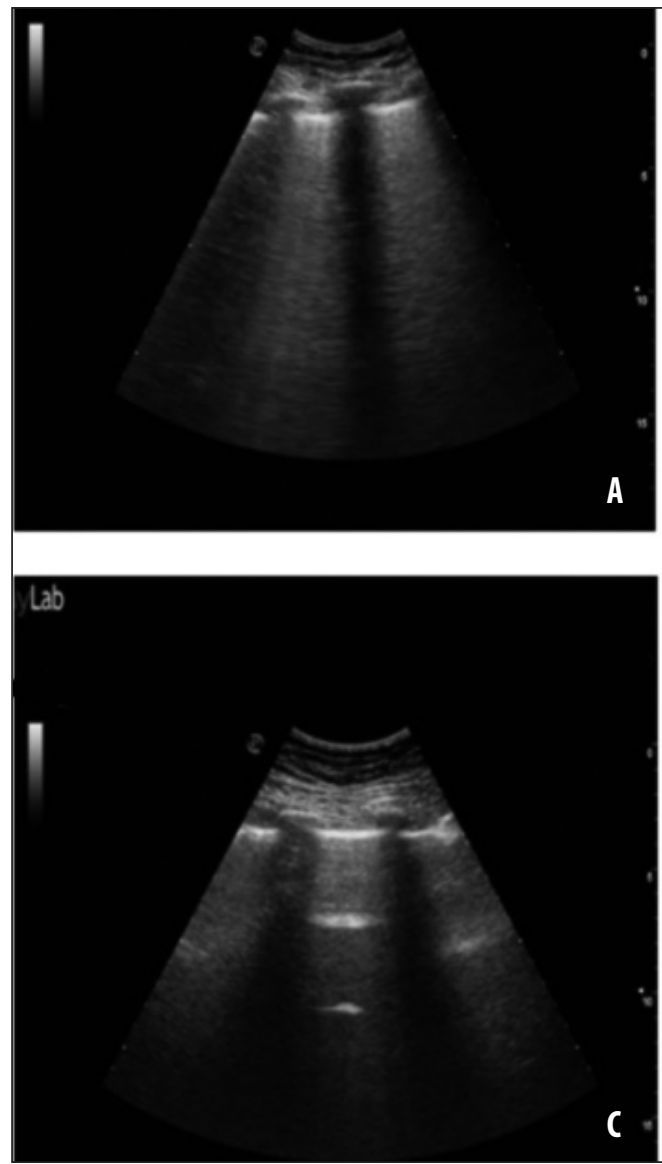
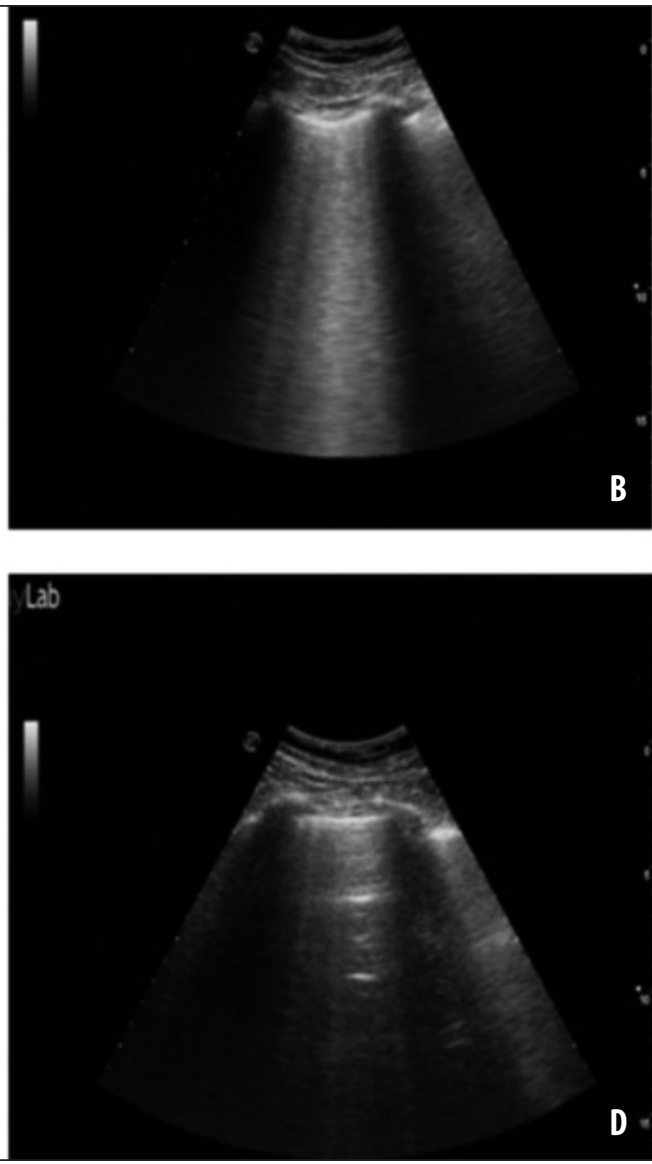

Fig. 2. Use of lung ultrasound to optimize mechanical ventilation parameters, including PEEP, in intensive care. Examination was performed in the 3rd intercostal space on midclavicular lines at 6 hour intervals, with clinical improvement after increase of PEEP.
Table I. Type and frequency of the main abnormalities detected by lung ultrasound

\begin{tabular}{cc}
\hline Lung ultrasound, $\mathbf{n}=\mathbf{1 3 5}$ & $\mathbf{n}(\%)$ \\
\hline Pleural line thickening & $121(89.6 \%)$ \\
\hline Focal B-lines & $21(15.5 \%)$ \\
\hline Multifocal B-lines & $110(81 \cdot 5 \%)$ \\
\hline Subpleural consolidations & $91(67.4 \%)$ \\
\hline Pleural effusion & $11(8.1 \%)$ \\
\hline
\end{tabular}

mechanical ventilation or for other reasons, posterior basal segments are visualized from most proximal lateral areas.

\section{When? Indications}

Interstitial syndrome, a classical feature of COVID-19 lung disease, causes gradual loss of lung aeration and build-up of fluid in the lungs. This is associated with evolving artifacts on ultrasound images, which may be used to assess progression of lung damage. At onset of disease lung ultrasound shows foci of interstitial syndrome, which may not be identifiable by X-ray yet, and appear as occasional 'ground glass' opacities on CT (Fig. 1B). As the disease progresses and the foci become more numerous, CT shows multiple 'ground glass' opacities, which correspond to multiple B-lines on ultrasound (Fig. 1C); further on the process becomes multifocal. Pulmonary exudation is associated with 'white lung' ultrasound pattern (Fig. 1D). Areas of alveolar consolidation may develop as well, which are identifiable by ultrasound too (Fig. 1E). As translobar consolidations develop, pulmonary tissue appears as a parenchymatous structure, possibly with static or dynamic air bronchograms, which may be assessed to optimize ventilation parameters and treatment strategy.

\section{RESULTS AND DISCUSSION}

In all symptomatic hospitalized patients assessed by lung ultrasound, abnormalities were present at least in one of the areas, mainly abnormal pleural line (121/89.6\%), pathologic B-lines (131/97\%), subpleural consolidations (91/67.4\%). A small proportion of patients had clinically insignificant pleural effusion (11/8.1\%). In all cases these abnormalities were bilateral. Mild disease was typically associated with ultrasound findings consistent with focal interstitial syndromes. Classical findings in more severe disease were multiple B-lines and pulmonary consolidations. Type and frequency of the main abnormalities detected by lung ultrasound are given in Table I.

Bilateral infiltrations are a classical CT finding in COVID-19 lung disease. These may appear several days before clinical deterioration, often at disease onset. In these cases X-ray findings do not reflect the changes present in the pulmonary tissue. We have noticed that both during disease progression and improvement, changes on X-ray are generally delayed, when compared to ultrasound findings, the latter being of higher diagnostic value in certain 

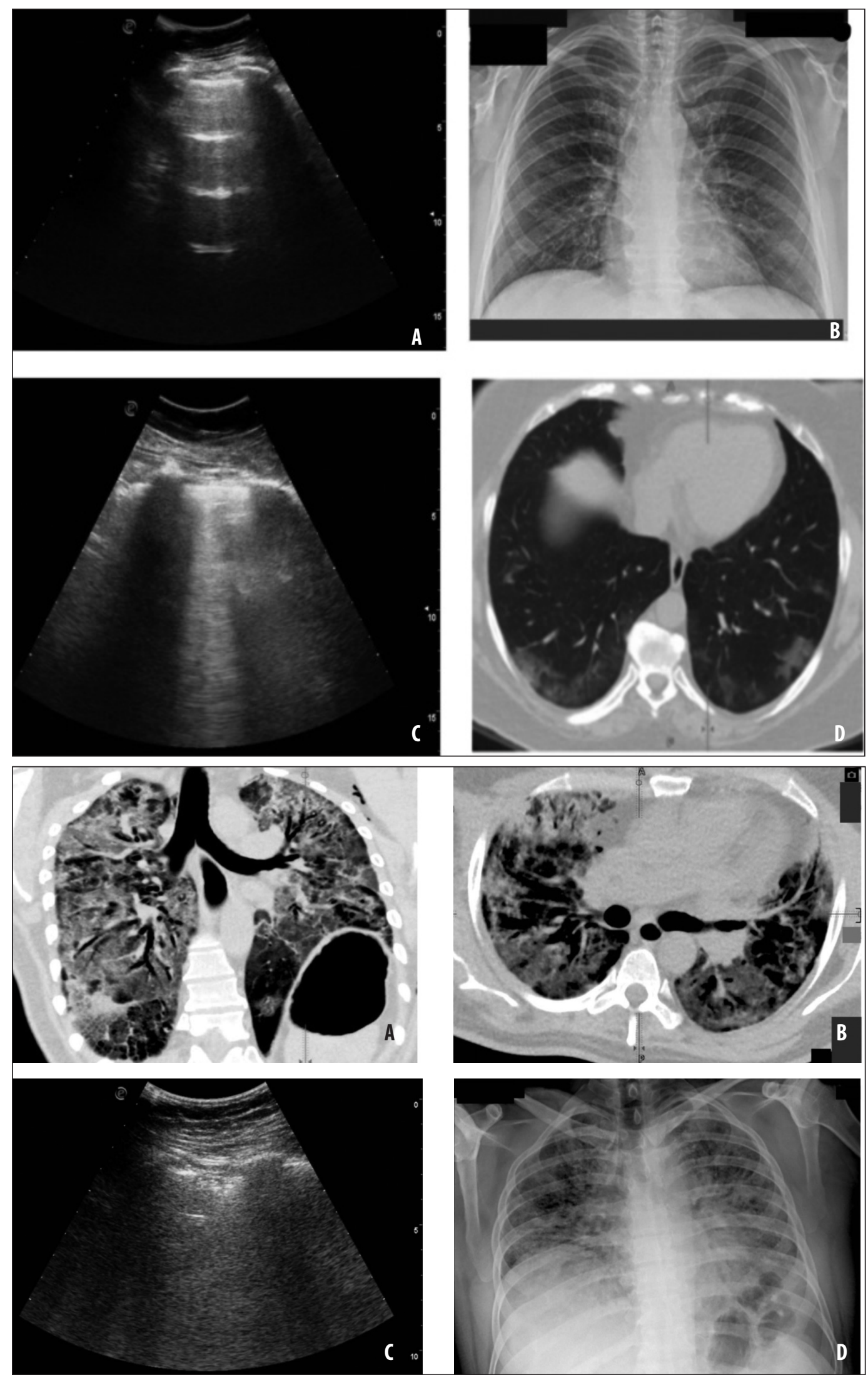

B

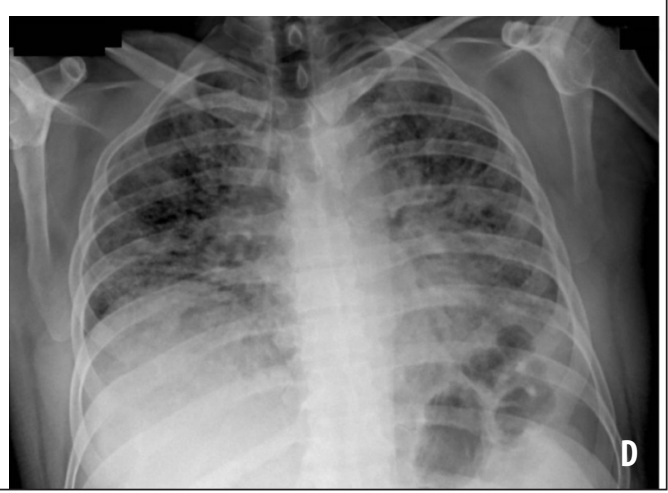

Fig. 3. Chest CT shows

marked changes in posterior and basal segments, which are not visible on chest $X$-ray. Lung ultrasound shows A-profile in ventral segments and merging B-lines with pleural thickening in posterior and basal segments. In mild COVID-19 cases lung ultrasound provides the necessary information about lung parenchyma without $X$-ray and CT.

Fig. 4. A, B. Over $80 \%$ of pulmonary parenchyma affected as shown by $\mathrm{CT}$; C. Ultrasonographic B-pattern, yet with occasional A-lines; D. Multisegmental bilateral foci on chest $X$-ray. circumstances. Below we present cases with no detectable abnormalities on chest X-ray despite typical lesions detected by CT and lung ultrasound (Fig. 3).

Lung ultrasound has also been used as a monitoring tool, especially in case of clinical deterioration. Clinical worsening was associated with progression of ultrasound findings over anterior points, which may be regarded as an adverse prognostic indicator. Also, in our experience, 

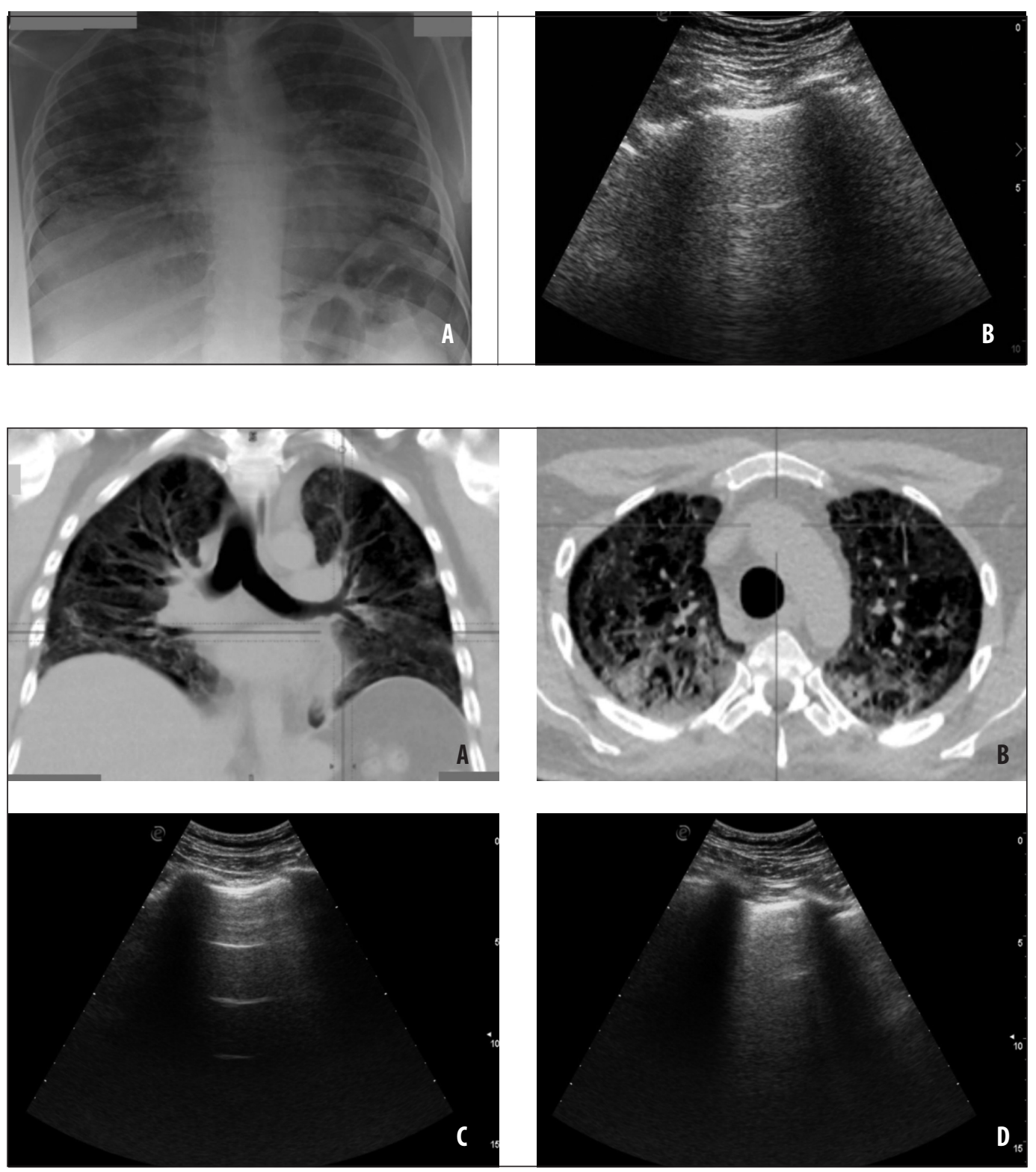

Fig. 5. A. X-ray showing improvement.

B. LUS score $=22$, A-lines appeared in ventral areas, and B-profile replaced the 'grey lung' (merging B-lines).
Fig. 6. A, B. Improvement on CT. C, D. LUS score improved to 10 points. optimized ventilatory support with adequate PEEP was associated with regression of alveolar consolidations and appearance of B-lines, signifying partial recovery of aeration. These changes were not always apparent on X-ray, and in some cases $\mathrm{X}$-ray showed deterioration, which did not correlate with clinical condition of patients.

We present a clinical case of a patient treated in the ICU. A male patient aged 45 was hospitalized 9 days after onset of illness with confirmed COVID-19 (PCR RNA SARSCoV-2+). SpO2 at time of admission was $96 \%$ on oxygen via face mask. In the ICU the patient was on intermittent non-invasive mechanical ventilation delivered by Mindray machine with FiO2 0.6: CPAP via Dräger NovaStar facial mask TS NIV MK/SE - size L, PEEP- 10 cmH2O, HFNC VeoFlo size L- $60 \mathrm{~L}$ - 3-4 hours per day (meals, hygiene, prevention of facial pressure sores from the mask). The patient spent at least 20 hours daily in prone position. The patient was stable. On day 12 of hospitalization the following findings were identified by lung ultrasound: A-pattern in anterior superior segments, B-pattern over all other points except for point 6 bilaterally, where merging B-lines were present. Total LUS score $=22$. $\mathrm{P} / \mathrm{F}$ ratio $=240 \mathrm{mmHg}$. The patient gradually deteriorated with $\mathrm{P} / \mathrm{F}$ ratio decreasing to $195 \mathrm{mmHg}$. On day 16 of hospitaization chest CT showed CORADS-6 (Fig. 4A). Lung ultrasound: LUS score $=26$ (Fig. 4B). Transthoracic echocardiography was performed in the modified prone position (right lateral decubitus swimmer position) to assess right chamber contractility. Apical 4-chamber view: dilated right chambers (right to left ventricle ratio 1:1). McConnell's sign. Right ventricular systolic pressure estimate by tricuspid regurgitation - 95 $\mathrm{mmHg}$. At 10:30 pm on the same day the patient's condition deteriorated, and respiratory acidosis developed ( $\mathrm{pH} 7.02$, pCO2 97 mmHg, pO2 60 mmHg, FiO2 90\%). Chest X-ray was performed (Fig. 4C).

Invasive mechanical ventilation was initiated via endotracheal tube 8.0, SIMV-PS (PEEP 14, PIP 16, FiO2 0.8). Consultation with ECMO team was obtained. With $80 \%$ of parenchyma affected and right ventricular insufficiency, ECMO was seen as indicated, and no contraindications 
were present, the sole limitation being the lack of staff. Ultrasound findings were considered (LUS 26), namely merging B-lines in 10 areas (except 2 areas where subpleural consolidations were present), but with occasional A-lines over the B-pattern suggesting partial aeration. PEEP was titrated to 20, PIP set to 16. Decision: ECMO stand-by, follow-up in 6 hours, and emergency initiation of ECMO in case of deterioration. On follow-up some improvement was noted. Echocardiography: RV to LV dimension ratio decreased, right ventricular systolic pressure $50 \mathrm{mmHg}$. LUS score improved to 22, A-lines appeared in ventral areas, and B-profile replaced the 'grey lung' (merging B-lines) (Fig. 5A, 5B).

After 52 hours of invasive mechanical ventilation the patient was extubated and switched to NIV: CPAP-therapy. On day 32 from the onset of illness the patient was in satisfactory condition, on $5 \mathrm{~L} / \mathrm{min}$ of nasal oxygen. Prior to discharge to rehabilitation, CT was obtained without contrast enhancement (Fig. 6A, 6B), and lung ultrasound was performed. LUS score $=10$ (Fig.6C, 6D).

In this case $\mathrm{X}$-ray based modalities were supplemented with regular ultrasound assessment, which was available 24/7. Lung ultrasound was crucial in hemodynamic assessment and respiratory support optimization, and helped avoid initiation of ECMO. Lung Ultrasound Score paralleled changes of the patient's condition throughout the treatment process, with the lowest grade of 10 registered prior to discharge, and the highest grade of 26 registered at time of ECMO stand-by. Closed survey of physicians at our unit showed an interesting difference between the extent of pulmonary parenchyma damage determined by radiological modalities versus ultrasound. 5 out of 6 intensive care specialists assessed radiological findings as more severe and were inclined to pessimistic prognosis.

\section{CONCLUSIONS}

The cases presented indicate potential advantages to the use of ultrasound in limited resource healthcare settings, especially with no access to CT or where the risk of transportation outweighs the value of information obtained. Grading of ultrasonographic findings in the lungs was sufficient for both initial assessment with identification of high risk patients, and routine daily monitoring. Hence, lung ultrasound may be used to predict deterioration, stratify risks and make clinical decisions. Ultrasonography may also be used to assess lung recruitability and ventilation potential, select appropriate modes of ventilation, monitor ventilated patients, and promptly detect complications. Ultrasound must become standard assessment in the setting of COVID-19 pandemics.

\section{REFERENCES}

1. Lichtenstein D, Goldstein I, Mourgeon E, Cluzel Ph, Grenier Ph, Rouby JJ. Comparative Diagnostic Performances of Auscultation, Chest Radiography, and Lung Ultrasonography in Acute Respiratory Distress Syndrome. Anesthesiol 2004; 100: 9-15.
2. Henschke Cl, Yankelevitz DF, Wand A, Davis SD. Shiau M Accuracy and efficacy of chest radiography in the intensive care unit. Radiol Clin North Am 1996; 34: 21-31.

3. Lichtenstein D, Peyrouset 0 . Is lung ultrasound superior to $\mathrm{CT}$ ? The example of a CT occult necrotizing pneumonia. Intensive Care Med 2006; 32: 334-335.

4. Yu CJ, Yang PC, Chang DB, Luh KT. Diagnostic and therapeutic use of chest sonography: value in critically ill patients. AJR Am J Roentgenol 1992; 159: 695-701.

5. Peng QY, Wang XT, Zhang LN. Chinese Critical Care Ultrasound Study Group (CCUSG). Finding of lung ultrasonography of novel coronavirus pneumonia during the 2019-2020 epidemic. Intensive Care Med. 2020; 46(5): 849-850.

6. Yang PC, Luh KT, Chang DB, Yu CJ, Kuo SH, Wu HD. Ultrasonographic evaluation of pulmonary consolidation. Am Rev Respir Dis 1992; 146: 757-62.

7. Lichtenstein D, Meziere G, Biderman P, Gepner A, Barre 0. The comettail artifact: An ultrasound sign of alveolar-interstitial syndrome. Am J Respir Crit Care Med 1997; 156: 1640-6.

8. Lichtenstein D, Meziere G, Biderman P, Gepner A. The comet-tail artifact: An ultrasound sign ruling out pneumothorax. Intensive Care Med 1999; 25: 383-8.

9. Lichtenstein D, Meziere $G$. A lung ultrasound sign allowing bedside distinction between pulmonary edema and COPD: The comet-tail artifact. Intensive Care Med 1998; 24: 1331-4.

10. Via G, Storti E, Gulati G, Neri L, Mojoli F, Braschi A. Lung ultrasound in the ICU: from diagnostic instrument to respiratory monitoring tool. Minerva Anestesiol 2012; 78 (11): 1282-96.

The work is carried out within the department's research work "COVID-19 in adults (clinical symptoms, aspects of pathogenesis, diagnostics, treatment)» (state registration number 0121U108048).

\section{ORCID and contributionship:}

Khrystyna Pronyuk: 0000-0003-2147-7599 A,E,F

Liudmyla Kondratiuk: 0000-0002-8855-5523 B,D,C

Andrii Vysotskyi: 0000-0002-6368-4361 B,D

Olga Golubovska: 0000-0003-3455-8718 A,D,E

Iryna Nikitina: 0000-0001-6595-2502 ${ }^{F}$

\section{CORRESPONDING AUTHOR}

\section{Khrystyna Pronyuk}

Infectious Diseases Department,

0.Bogomolets National Medical University, Kyiv, Ukraine

tel: +380503712348

e-mail:khrystynapronuk@gmail.com

Received: 22.04 .2021

Accepted: 29.07 .2021

A - Work concept and design, B - Data collection and analysis, C - Responsibility for statistical analysis,

D-Writing the article, $\mathbf{E}$-Critical review, $\mathbf{F}$ - Final approval of the article 